\title{
ABOUT SIMULATION MODELING OF HM-QUEUEING NETWORK WITH UNRELIABLE SYSTEMS
}

\author{
Mikhail Matalytski ${ }^{l}$, Sviataslau Statkevich ${ }^{2}$ \\ ${ }^{1}$ Institute of Mathematics, Czestochowa University of Technology, Poland \\ ${ }^{2}$ Faculty of Mathematics and Computer Science, Grodno State University, Belarus \\ m.matalytski@gmail.com,sstat@grsu.by
}

\begin{abstract}
The Markov network with unreliable queueing systems and incomes are considered. Analytical results on research of exponential networks in a case when incomes of transitions between network states depend on time are reduce. The algorithm of simulation modeling of HM-queueing networks based on the 0-moments. This method allows one to find incomes of networks when the service time of messages, serviceable work time of channels and restoration time of failure channels has any distribution. Results of simulation modeling are compared to analytical results. High accuracy of the developed algorithm is shown.
\end{abstract}

\section{Introduction}

At the Markov queueing network with incomes or HM (Howard-Matalytski)network (QN) a messages at transitions from one queueing system (QS) to another bring the last QS some income, and income of the first system decreases for the same value. Such networks can be used as stochastic models incomes forecast in systems of interbank payments, in the insurance companies and logistical transport systems, at the enterprises from realization of their production, and also at an estimation of expenses on the maintenance flexible computing cluster [1].

Let us examine open exponential QN with one type messages which consist of $n$ queueing systems (QS) $S_{1}, S_{2}, \ldots, S_{n}$. The Poisson flow of one type messages with arrival rate $\lambda$ comes into network from outside medium - system $S_{0}$. Let the system $S_{i}$ will consist of $m_{i}$ identical service channels, the service time in each of which has exponential distribution with parameter $\mu_{i}, i=\overline{1, n}$.

Let's suppose, that service channels of system $S_{0}$ are absolutely reliable. At the other QS $S_{1}, S_{2}, \ldots, S_{n}$ service channels are exposed to random failure and serviceable work time of each channel of system $S_{i}$ has exponential distribution with parameter $\beta_{i}, i=\overline{1, n}$. After failure the service channel immediately starts to be restored and restoration time also has exponential distribution with parameter $\gamma_{i}$, 
$i=\overline{1, n}$. Let's consider that service time of messages, durations of serviceable work of channels and restoration time of service channels are independent random variables. The state of such network could be described via vector

$$
z(t)=(d(t), k(t))=\left(d_{1}, d_{2}, \ldots, d_{n}, k_{1}, k_{2}, \ldots, k_{n}, t\right),
$$

where $d_{i}(t)$ - number of serviceable channels in system $S_{i}$ at the moment $t$, $0 \leq d_{i}(t) \leq m_{i}, k_{i}$ - messages number in system $S_{i}$ at the moment $t, t \in[0,+\infty)$, $i=\overline{1, n}$. Let $p_{0 j}$ - probability of message enters from outside to the system $S_{j}$, $\sum_{j=1}^{n} p_{0 j}=1 ; p_{i j}$ - probability of message transitions from system $S_{i}$ to the system $S_{j}, \sum_{j=0}^{n} p_{i j}=1, i=\overline{1, n}$. Matrix $P=\left\|p_{i j}\right\|_{(n+1) \times(n+1)}$ is matrix of passage probabilities of irreducible Markov chains. Let's assume that if the service channel would fail while completing some message, then after the restoration the interrupted message will be completed. Message during its transition from system $S_{i}$ to system $S_{j}$ brings to system $S_{j}$ some income and income of system $S_{i}$ descend on this value correspondently, $i, j=\overline{0, n}$. Service rate of message occurs according to discipline FIFO.

In the work [2] the system of difference-differential equations (DDE) for the expected incomes of QS is received. For closed QS when $\lambda=0, p_{0 i}=p_{i 0}=0$, $i=\overline{1, n}$, the system becomes

$$
\begin{aligned}
& \frac{d v_{i}(d, k, t)}{d t}=-\sum_{j=1}^{n}\left[\mu_{j} \min \left(k_{j}, d_{j}\right)+\beta_{j} d_{j}+\gamma_{j}\left(m_{j}-d_{j}\right)\right] v_{i}(d, k, t)+ \\
& \quad+\sum_{j=1}^{n}\left[\beta_{j} d_{j} v_{i}\left(d-I_{j}, k, t\right)+\gamma_{j}\left(m_{j}-d_{j}\right) v_{i}\left(d+I_{j}, k, t\right)\right]+ \\
& +\sum_{\substack{c, s=1 \\
s \neq c}}^{n} \mu_{s} \min \left(k_{s}, d_{s}\right) p_{s c} v_{i}\left(d, k+I_{c}-I_{s}, t\right)+R_{i j c s}^{(1)}(d, k, t), i=\overline{1, n}
\end{aligned}
$$

where

$$
\begin{gathered}
R_{i j c s}^{(1)}(d, k, t)=\sum_{j=1}^{n}\left[\gamma_{j}\left(m_{j}-d_{j}\right) q_{i j}\left(d+I_{j}, k, t\right)-\beta_{j} d_{j} g_{i j}\left(d-I_{j}, k, t\right)\right]+ \\
+\sum_{\substack{c, s=1 \\
c \neq s}}^{n} \mu_{s} \min \left(k_{s}, d_{s}\right) p_{s c} r_{i c s}\left(d, k+I_{c}-I_{s}, t\right)+r_{i}(d, k) .
\end{gathered}
$$


and $q_{i j}\left(d+I_{j}, k, t\right)$ - income of QS $S_{i}$, when the service channel in the system $S_{j}$ is restored, $i=\overline{1, n} ; g_{i j}\left(d-I_{j}, k, t\right)$ - income of QS $S_{i}$, when the service channel in the system $S_{j}$ is failure, $i=\overline{1, n} ; r_{i c s}\left(d, k+I_{c}-I_{s}, t\right)$ - income of QS $S_{i}$, when message after servicing in system $S_{s}$ transfer to system $S_{c}, i, c, s=\overline{1, n}, c \neq s$; $r_{i}(d, k)$ - income of QS $S_{i}$ in unit of time.

Usually failure or restoration of service channels in system $S_{i}$ are not depended with their breakages or restoration in others QS, then

$$
\begin{aligned}
& q_{i j}\left(d+I_{j}, k, t\right)=\left\{\begin{array}{l}
q_{i}\left(d+I_{j}, k, t\right), i=j, \\
0, \quad i \neq j,
\end{array}\right. \\
& g_{i j}\left(d-I_{j}, k, t\right)= \begin{cases}g_{i}\left(d-I_{j}, k, t\right), i=j, \\
0, & i \neq j .\end{cases}
\end{aligned}
$$

and

$$
\begin{aligned}
R_{i j c s}^{(1)}(d, k, t) & =R_{i c s}^{(1)}(d, k, t)=\gamma_{i}\left(m_{i}-d_{i}\right) q_{i}\left(d+I_{i}, k, t\right)-\beta_{i} d_{i} g_{i}\left(d-I_{j}, k, t\right)+ \\
& +\sum_{\substack{c, s=1 \\
c \neq s}}^{n} \mu_{s} \min \left(k_{s}, d_{s}\right) p_{s c} r_{i c s}\left(d, k+I_{c}-I_{s}, t\right)+r_{i}(d, k) .
\end{aligned}
$$

The system of the equations (2) can be reduced to systems of the linear nonhomogeneous ordinary differential equations with a constant coefficient which in the matrix form can be written as

$$
\frac{d D_{i}(t)}{d t}=A D_{i}(t)+Q_{i}(t)
$$

where $D_{i}^{T}(t)=\left(v_{i}(1, t), v_{i}(2, t), \ldots, v_{i}(L, t)\right)$ - vector of the expected incomes of system $S_{i}, L$ - number of network states. The system (3) can be solved a direct method (by means of matrix exponent) or a method of Laplace transformations.

In the given article main principles of simulation modeling of HM-queueing network with unreliable systems are described. The service time of messages, the serviceable work time of channels and the restoration time of breakage channels have a arbitrary laws of distribution. For an accuracy estimation in exponential case the results received by means of simulation modeling are compared to the analytical results. 


\section{Simulation modeling of HM-queueing networks}

The simulation model of QN represents the computer program reproducing the process of functioning of the network directed on reception of a vector of states (1) during some discrete moments of time $t_{l}, l \geq 0$. At modeling the initial condition of the network at the moment of time $t_{0}$ is specified. Then according to the mathematical model the trajectory of the vector of states on time intervals $\left[t_{0}, t_{1}\right)$, $\left[t_{1}, t_{2}\right) \ldots$ is under construction. The moments of time $t_{l}$ correspond to the moments of receipt of the messages from on outside medium, message leaving in an outside medium, to the termination of service of the message in QS, failure of service channels, or restoration of faulty channels. For example, the message pass from system $S_{i}$ to system $S_{j}$, then $(n+i)$ th component of vector of state (1) decreases on 1 and $(n+j)$ th increase on $1, i, j=\overline{1, n}$. If there is a breakage of a service channel in system $S_{k}$ then $k$-th component of vector of states decreases on 1 , in the case of restoration - increases on 1 . In a time interval $\left[t_{l-1}, t_{l}\right)$ changes do not occur. The moments of time $t_{l}$ are called 0 -moment.

Thus, each 0 -moment contains the information about which event has occurred: the message arrival from the outside medium, the termination of service of the message, breakage or restoration of service channel, from what QS transition as a result of end of service of the message, in which QS there was a restoration of a service channels or breakage and number of this channel, and also the moment of time of approach of it of events is carried out.

Let's assume that expected incomes of systems and a network depending on a vector of states vary as follows: at transition of the message from $i$-th QS in $j$-th, the second QS increases the income by a random variable (RV) $r_{i j}$ with distribution function $F_{1 i}(x)$, and the first QS reduces the income on same RV. If the network does not make any transitions between states, each system $S_{i}$ receives the income $r_{i}$ for a unit of time during all period of stay of a network in a current state where $r_{i}$ is RV with distribution function $F_{0 i}(x)$. In the case when in $i$-th tQS the service channels is breakage its income decreases on RV $g_{i}$ with distribution function $G_{i}(x)$; if the service channel is restored, income $i$-th QS decreases on RV $q_{i}$, having the distribution function $Q_{i}(x), i=\overline{1, n}$.

At modeling by initial data will be: network type - open or closed, number of network QS $n$, matrix of passage probabilities $P$, initial distribution of messages in queues of network systems, number of service channels $m_{i}$ and number of serviceable channels $d_{i}$ in network systems, $i=\overline{1, n}$, laws of distribution of servicing 
times of messages, laws of distribution of times of serviceable work of channels and restoration faulty values of incomes $r_{i}, r_{i j}, g_{i}, q_{i} i=\overline{1, n}$, and their laws of distribution.

Let's consider each step of the algorithm of a finding of expected incomes for a network with an unreliable QS.

0 . Generation of initial network state at the time $t=0$. The set initial data of a modeled network are analyzed. The 0-moments which describe the moments of the termination of service of some messages, the moments of failure of service channels and the moments of restoration of faulty service channels are created.

1. Receiving of the 0-moment. All actions connected with transition of the messages, by loading of others QS, breakage of service channels and restoration faulty, calculation of new values of incomes caused by these changes are carried out. We will list them.

2.1. If on this step to one of the service channels of QS with number $i, i=\overline{1, n}$, there was a breakage:

- time during which the service channel will be restored according to the set law of distribution is modeled;

- the moment of the termination of restoration is added in the general queue of events of a network;

- income $i$-th QS decreases on value $g_{i}$;

- if during this moment the service channel has been occupied by service of the message,

- then the message becomes in the beginning of queue of expectation of service in this QS:

- afterservice time for the given message pays off as the difference between length of an interval of time which the current message should be served, and length of an interval of time in which the current was actually serviced;

- afterservice time the given message is remembered.

2.2. If on the given step there comes the event connected with the termination of restoration of a service channel in QS with number $i, i=\overline{1, n}$, then:

- time when the given service channel will fail again according to the set law of distribution is modeled;

- breakage time is added in the general queue of events of a network;

- income $i$-th QS decreases on value $q_{i}$;

- if in queue of $i$-th QS there are the messages expecting service then service of the following message begins.

2.3. If on the given step in one of service channel of QS with number $j$ service of message has ended then this message according to a matrix of passage probabilities transfer to QS with number $i, i, j=\overline{1, n},(i \neq j)$ and:

- if all service channels of $i$-th QS are occupied, 
- then we place the message in queueof this QS,

- else the message starts to be served by a free channel,

- the message holding time is modeled according to the law of distribution with the parameters corresponding to these QS;

- time of the end of service of the message is remembered; income $i$-th QS increases on value $R_{i j}$, and $j$-th decreases for the same value;

- further we pass to consideration of QS which has finished servicing the message:

- if in queue of QS with number $j$ there are still messages,

- then is taken the first message from queue and begins to be served by a free serviceable channel; the moment of the termination of this service is located in the general queue of events of a network.

3. We carry out transition to a step 1 .

Modeling of work of QN on some interval of time is made several times. As a result for each experiment the random trajectory of change of incomes in time turns out. For a reception of the resultant of trajectory for the expected income of network, trajectories received as result of imitating experiments are averaged.

\section{Examples}

Example 1. Let's consider a closed QN with unreliable systems and central QS: $n=4, K=14, S_{1}, S_{2}, S_{3}$ - peripherial QS, $S_{4}$ - central QS. Number of service channels: $m_{1}=4, m_{2}=7, m_{3}=3, m_{4}=5$. Service time of messages in system $S_{1}$ has exponential distribution with parameter $\mu_{1}=0.5 ; S_{2}$ - normal distribution with parameters $a_{2}=3, \sigma_{2}=0.3 ; S_{3}$ - even distribution on [0,5]; $S_{4}$ - exponential distribution with parameter $\mu_{4}=2$. Serviceable work time of channels in system $S_{1}$ - exponential distribution with parameter $\beta_{1}=1 ; S_{2}$ - normal distribution with parameters $a=1, \sigma=0.5 ; S_{3}$ - even distribution on $[0,1.5] ; S_{4}$ - exponential distribution with parameter $\beta_{3}=2$. Restoration time of failure channels in systems: $S_{1}$ exponential distribution with parameter $\gamma_{1}=2 ; S_{2}$ - normal distribution with parameters $a=1.9, \sigma=0.9 ; S_{3}$-even distribution on $[0,4] ; S_{4}$ - even distribution on $[0,4.8]$. Probabilities of transitions: $p_{14}=p_{24}=p_{34}=1, p_{41}=p_{42}=p_{43}=1 / 3$, other $p_{i j}=0, i, j=\overline{1,4}$. The vector of states during the initial moment of time looks like $(3,6,2,4,3,3,4,4)$. Incomes of systems $S_{i}, i=\overline{1,4}$ in a unit of time: $r_{1}=r_{2}=1, r_{3}=1.5, r_{4}=3$. Incomes of transitions between network states: $\quad R_{1 j}(t)=-0.3+0.5 \cos (0.5-1.7 t), \quad R_{2 j}(t)=132+32 \cos (19.5+0.48 t)$, 
$R_{3 j}(t)=139.7-12.7 \sin \left(\frac{2 \pi}{3}+3 t\right), R_{4 j}(t)=15-\sin \frac{\pi}{2}, j=\overline{1,4}$. In case of breakage of service channels each of network systems sustains losses in size: $g_{1}(t)=\cos t+1$, $g_{2}(t)=\cos t+2, g_{3}(t)=2 \cos t+1, g_{4}(t)=2 \cos t+2$, in case of restoration of service channels $q_{1}(t)=\sin t+1, q_{2}(t)=\sin t+3, q_{3}(t)=q_{4}(t)=3 \sin t+0.5$. Let, also, during the initial moment of time the income of all QS is equal to 0 .

In Figure 1 change of expected incomes of a network QS on time interval $[0,10]$ are resulted.

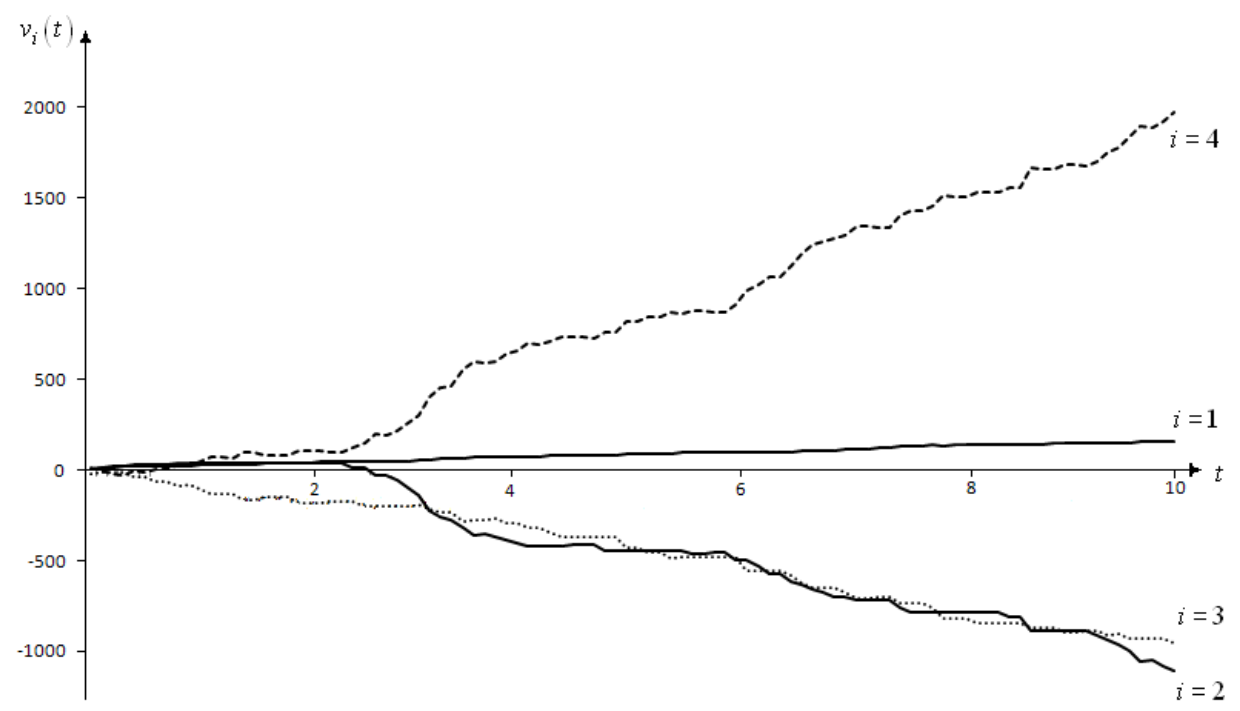

Fig. 1. Changes of expected incomes of network systems

Example 2. Let's consider a closed exponential QN with unreliable systems and central QS: $n=3, K=4, m_{i}=1, i=\overline{1,3}$. The number of states of such network equals 180. Let $\mu_{1}=\mu_{2}=1, \mu_{3}=2, \beta_{1}=1.3, \beta_{2}=0.5, \beta_{3}=1.9, \gamma_{1}=0.5$, $\gamma_{2}=0.2, \gamma_{3}=1.7$. Probabilities of transitions: $p_{13}=p_{23}=1, p_{31}=2 / 5, p_{32}=3 / 5$, other $p_{i j}=0, i, j=\overline{1,3}$. Incomes of transitions between network states: $r_{i}=0.02$, $R_{1 j}(t)=e^{-3 t}+3, \quad R_{2 j}(t)=e^{t} \sin t+2, \quad R_{3}(t)=3-\sin t, \quad g_{1}(t)=e^{t}+5$, $g_{2}(t)=2 e^{-t}, g_{3}(t)=-\cos t+0.5, q_{1}(t)=q_{2}(t)=q_{3}(t)=0.9$. The vector of states during the initial moment of time looks like $(1,1,1,1,1,2)$. Also, let the initial moment of time the income of all QS is equal to 0 .

For such a network by the decision of system of DDE (2), received the decision for expected incomes of network systems. By means of simulation modeling expected incomes also have been calculated and compared to analytical results. In 
Figure 2 graphics of behaviour of the expected income for central system $S_{3}$ on an interval of time $[0,10]$, calculated are resulted in both methods. The dashed line represents a trajectory of change of the expected income received by means of simulation modeling, continuous - by the decision of system of DDE (2). Apparently from the Figure 2, the graphics differ slightly. Thus the simulation model possesses high accuracy.

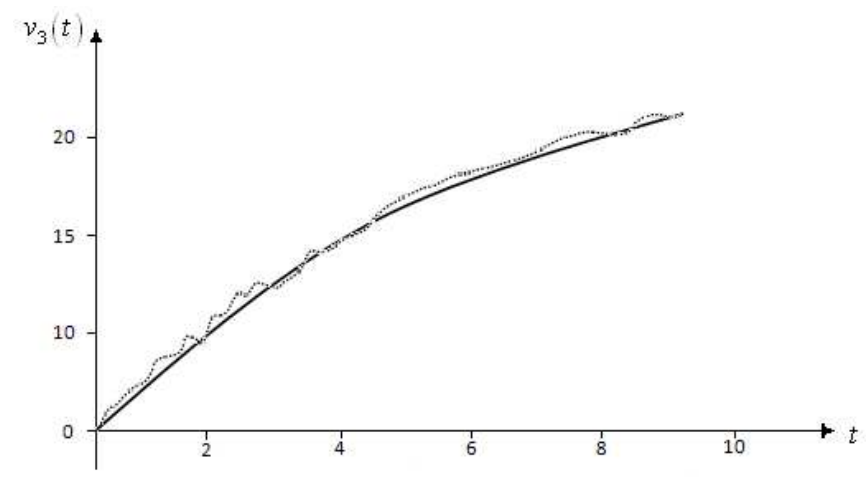

Fig. 2. Changes of expected incomes of central QS $S_{3}$

\section{Conclusions}

In the article the developed simulation model of HM-queueing network with unreliable systems is described. By comparison with exact analytical results in exponential case, received by the decision of system of the different-differential equations for expected incomes, it is shown, that the constructed simulation model possesses enough high accuracy.

\section{References}

[1] Matalytski M.A., On some results in analysis and optimization of Markov networks with incomes and their application, Automation and remote Control 2009, 70, 10, 1683-1697.

[2] Statkevich S.E., Matalytski M.A., Investigation of HM-network with unreliable queueing systems, Westnik GrSU, 2010, 2, 31-40 (in Russian). 